\title{
Mechanical properties of the spinal cord and brain: comparison with clinical-grade biomaterials for tissue engineering and regenerative medicine
}

Richard D Bartlett ${ }^{1,2,3}$, Despoina Eleftheriadou ${ }^{1,2}$, Rachael Evans ${ }^{1,2}$, David Choi ${ }^{1,3,4}$, James B Phillips ${ }^{1,2^{*}}$

${ }^{1}$ Centre for Nerve Engineering, University College London, London, WC1E 6BT, United Kingdom

${ }^{2}$ Department of Pharmacology, UCL School of Pharmacy, University College London, WC1N 1AX, United Kingdom

${ }^{3}$ Brain Repair and Rehabilitation, Institute of Neurology, University College London, WC1N $3 B G$

${ }^{4}$ Victor Horsley Department of Neurosurgery, National Hospital for Neurology and Neurosurgery, Queens Square, London, United Kingdom, WC1N $3 B G$.

* Correspondence: James B Phillips, Department of Pharmacology, UCL School of Pharmacy, University College London, WC1N 1AX, United Kingdom. Email: jb.phillips@ucl.ac.uk. Tel: 02077535570.

\section{KEY WORDS:}

Biomechanics; dynamic mechanical analysis; biomaterials; tissue engineering; central nervous system; hydrogels 


\section{ABSTRACT:}

Disorders affecting the central nervous system are a leading cause of disability in the world. Regenerative medicine using biomaterial-based therapies is a growing field that has potential application in the areas of spinal cord injury, neurodegenerative disorders and stroke. The mechanical properties of biomaterials implanted into the central nervous system are critical for effective integration with host tissue, but the biomechanical properties of the host tissue remain poorly characterised and assessing the stiffness of both soft biomaterials and central nervous system tissue remains challenging. Here, we describe a bespoke mechanical characterisation method that facilitates robust measurement of fresh spinal cord and brain tissue and allows direct like-for-like mechanical benchmarking for matching of clinical-grade hydrogels suitable for regenerative medicine. We report differences in the mechanical properties of spinal cord tissue dependent on anatomical origin, regional variations in brain tissue stiffness, and quantify the extent of mechanical anisotropy within the cervical spinal cord. We then demonstrate that the mechanical properties of clinical-grade collagen, fibrin and alginate hydrogels can be tuned to closely mimic the mechanical properties of different regions within the central nervous system. 


\section{MAIN TEXT:}

\section{Introduction}

Disorders affecting the central nervous system (CNS) are a leading cause of disability worldwide [1]. Spinal cord injury affects between 250,000 and 500,000 new people each year [2] and has an estimated worldwide cost between \$16 - \$26 billion per annum [3], whilst neurodegenerative disorders such as Parkinson's and Alzheimer's disease contribute to $28 \%$ of all years lived with disability and their prevalence is only expected to increase with an ageing population [4]. The consequences of damage to the CNS are severe and the effect on patient quality of life is significant. Despite this, there remains a lack of effective disease-modifying therapies.

Regenerative medicine has recently begun to offer new paradigms in the treatment of CNS disorders, with many regenerative strategies underpinned by biomaterial-based approaches [5]. Biomaterials have been used to enhance the survival of therapeutic cells transplanted into the CNS [6], provide essential structural or mechanical support [7], and act as depots to enable local drug or gene therapy delivery $[8,9]$. Consequently, they have enabled considerable progress in the development of potential new treatments for a range of CNS disorders, including spinal cord injury [10], stroke [11], traumatic brain injury [12], and neurodegenerative diseases such as Alzheimer's and Parkinson's [13, 14]. Indeed, the structural support provided by biomaterials may be essential for facilitating effective axonal regrowth after human spinal cord injury [15], and the combination of biomaterial-based approaches with cell therapies has been used to ameliorate neurodegeneration in animal models of Parkinson's disease $[16,17]$. 
Mechanical properties are an essential consideration for any biomaterial implanted into the CNS. Stiffness of the local tissue microenvironment has been implicated in a range of cell behaviours and is therefore critical for determining the effectiveness of biomaterial-based regenerative medicine strategies. Firstly, the mechanical properties of a biomaterial determine the host tissue response. Astrocytes are highly mechanosensitive and poorly matched mechanical properties cause cell hypertrophy, upregulation of GFAP and a phenotypic response similar to that seen after CNS injury $[18,19]$. Such changes are undesirable in the context of a neuroregenerative therapy and therefore appropriately matched mechanical properties between the host tissue and implant is critical [20]. Secondly, mechanical properties may influence the response and phenotype of therapeutic cells. For example, stiffness has been shown to alter the differentiation, adhesion and migration of stem cells [21-24]. The mechanical properties of substrate or tissue also influence neuronal behaviour, including the rate of neurite extension [25], direction of axonal outgrowth and the extent of neurite branching $[26,27]$. Thirdly, at the macroscopic level, any undue forces occurring at the biomaterial-tissue interface will disrupt structural continuity and hinder traversing cells or axons. Mechanical integration with host tissue is, therefore, an essential consideration for any biomaterial-based therapy aimed at promoting CNS repair.

Moreover, changes in the host tissue mechanical properties of CNS upon injury may have significant consequences for neuronal regeneration. These have not so far been fully characterised [28]. However, in contrast to other parts of the body where scar tissue is traditionally stiffer than the surrounding healthy tissue [28, 29], recent data have indicated that CNS tissue may in fact soften after injury but display increased viscosity $[30,31]$. Whether these softening changes are retained 
over time remains unclear though, and chronic CNS injury may still be associated with tissue stiffening [32].

Despite this, the mechanical properties of biomaterials for CNS repair remain poorly characterised. This is primarily due to the difficultly in robustly quantifying the mechanical properties of CNS tissue and then directly comparing this to biomaterials for CNS repair [33]. Viscoelastic behaviours mean that traditional mechanical testing paradigms such as pull to failure or ramp tests provide only limited information about the stiffness of tissues. Similarly, single values for tissue modulus may not be fully representative of the overall tissue response due to stress-stiffening behaviours. Techniques such as atomic force microscopy (AFM) provide excellent spatial resolution, yet they involve substantial tissue processing and only provide data about highly localised mechanical properties on the surface of a specimen. This makes them unsuited for screening the overall bulk mechanical properties of a tissue or for the direct benchmarking of biomaterials used in regenerative medicine. Similarly, rheometry allows precise mechanical characterisation of materials such as hydrogels, yet it only provides information about shear modulus and is less suited for testing heterogeneous fresh biological tissues.

Here, we outline a novel protocol using a bespoke frequency-dependent dynamic testing paradigm specifically developed for characterising the bulk mechanical properties of fresh CNS tissue. To develop this, we exploited the advantages offered by dynamic mechanical analysis (DMA). This is a versatile and flexible, automated technique that utilizes sinusoidal compression to map the mechanical properties of soft viscoelastic materials, including biological specimens. It provides information for both the elastic and viscous components of the biomaterial, through the measurement of phase-lag relative to the original 
compressive or tensional movement. Using this, we perform comprehensive mechanical characterisation of different spinal cord and brain regions, and provide proof of principle that the same methodology can be applied to comprehensively 'benchmark' the mechanical properties of clinical-grade biomaterials suitable for use in regenerative medicine.

\section{Methods}

\section{Tissue harvest}

Male Sprague-Dawley rats weighing approximately $200 \mathrm{~g}$ were culled using pentobarbital in accordance with UK Home Office Schedule 1 regulations. For spinal cord, a dorsal mid-line incision was made from head to tail and the underlying spinal musculature was exposed. The thoracolumbar fascia on either side of the spinal column was then pierced, and blunt dissection was used to create two para-spinal openings. The ribs, surrounding musculature and connective tissue were then dissected to isolate the spinal column. Transverse cuts were made just below the base of the skull and above the sacrum to remove the spinal column in its entirety.

A syringe expulsion technique was then used to rapidly remove the spinal cord from the spinal canal [34]. Briefly, a blunt syringe was overlaid with a trimmed Gilson P200 tip and inserted into the caudal end of the spinal canal. It was then pushed until the most caudal lumbar vertebrae was heard to audibly fracture, thus ensuring that a water-tight seal had been formed. Approximately $5-10 \mathrm{~mL}$ of $\mathrm{AQIX}^{\circledR}$ RS-I biopreservation media was then rapidly injected to expel the spinal cord cleanly from the cervical end of the spinal canal. Tissue was then delicately run over a micro-spatula to remove any residual connective tissue or dura. Samples were immediately placed in ice-cold AQIX ${ }^{\circledR}$ RS-I solution ready for 
mechanical testing or in cold $4 \%(\mathrm{v} / \mathrm{v})$ paraformaldehyde for histological analysis.

For brain tissue, the head was decapitated and skin removed. Friedman rongeurs were used to remove underlying musculature and fascia. Bone cutters were then used to make a midline incision from the base of skull along the sagittal suture to the nose. The whole brain was removed intact and placed in ice-cold AQIX ${ }^{\circledR}$ RS-I solution. The specimen was then carefully dissected under a stereomicroscope. For cortex, dura and surface blood vessels were delicately removed using microforceps. For corpus callosum, a micro-spatula was gently placed in the longitudinal fissure to separate cortices and the tip of a No. 12D carbon steel scalpel blade (Swann-Morton) used to dissect the corpus callosum from surrounding deep brain structures. Tissue was kept in ice-cold AQIX ${ }^{\circledR}$ RS-I solution during the dissection process and until the point of geometric measurement and mechanical testing.

\section{Sample preparation and geometric measurement}

Following syringe extraction, spinal cord tissue was prepared for mechanical testing. Cord tissue was cut using two crossed No. 11 scalpel blades (Swann Morton) to generate either cervical, thoracic and lumbar sections approximately 5 $\mathrm{mm}$ in length. These were then geometrically assessed using a Canon EOS camera with dedicated macro lens. Height, length and width measurements were then extracted using ImageJ and geometric values entered into the DMA WinTest 7 software to determine pre-strain and dynamic mechanical amplitude for each individual sample. The same process was applied for brain and biomaterial specimens. WinTest7 allows specimens to be modelled as either a cylinder, square or rectangle. Radial sections of spinal cord tissue were modelled within WintTest 7 as a rectangle, as when viewed in cross-section they more closely resembled a rectangular profile than a cylindrical profile. Likewise, sections of 
corpus callosum were modelled as rectangular. Longitudinal spinal cord sections, cortex and all hydrogels were modelled as cylinders. Parameters were inputted into WinTest 7 before pre-strain was applied and changes to specimen geometry were then automatically accounted for within the WinTest 7 software.

\section{Dynamic mechanical analysis}

After geometric dimensions had been determined, samples were transferred to a Bose Electroforce 3200 (TA Instruments, DE, USA) for compressive dynamic mechanical analysis (DMA). Spinal cord sections were placed between two $10 \mathrm{~mm}$ metal platens; load and displacement values were zeroed. Platens were then brought together at the slowest possible speed until a small initial deflection in platen displacement was observed using the WinTest 7 software, indicating that sample-platen contact had occurred. An initial ramp of $15 \%$ pre-strain was applied to ensure robust sample contact and a $1 \mathrm{~Hz}$ pre-conditioning cycle performed to minimise inter-sample variability [35]. A measured DMA cycle was then performed using $2 \%$ dynamic amplitude and a $1-70 \mathrm{~Hz}$ ascending frequency sweep. Once the sweep had been completed, a repeat $1 \mathrm{~Hz}$ validation measurement was conducted to assess for any signs of permanent mechanical deformation. Likewise, height measurements were repeated to check for evidence of geometric change. All samples were tested at room temperature $\left(21^{\circ} \mathrm{C}\right)$ and fully hydrated (100\% humidity) using AQIX ${ }^{\circledR}$ RS-I media throughout preparation and testing. A schematic overview of the method used is detailed in Figure 1 and key terms used in deformation mechanics are summarised in 


\section{Information Box 1.}

(a)
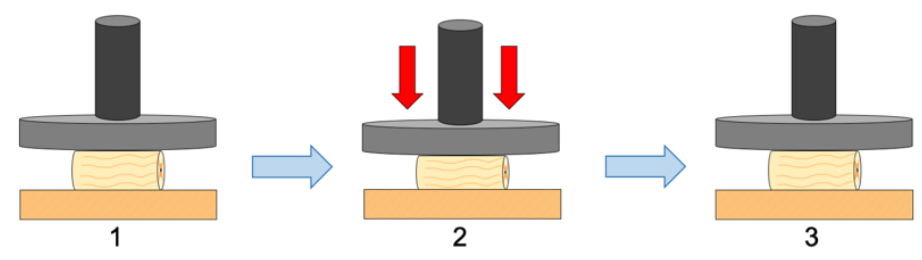

(b)

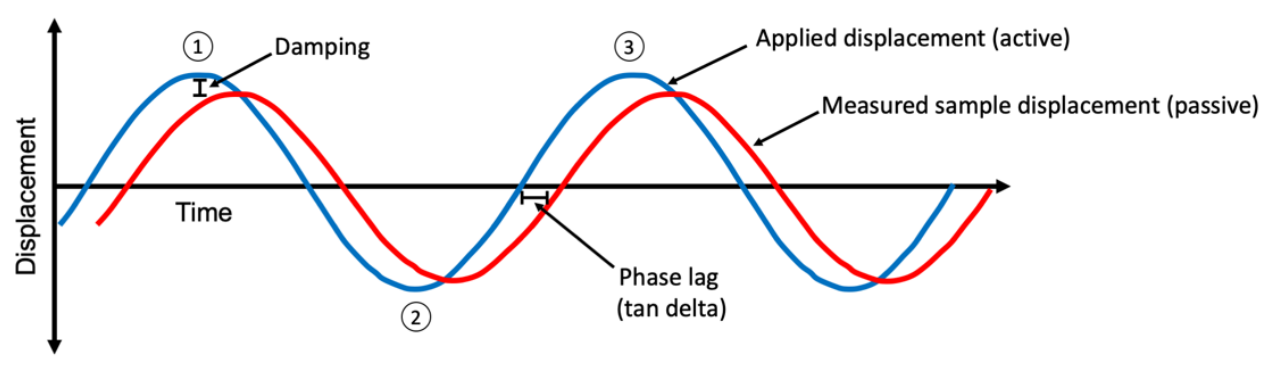

Figure 1. Overview of the mechanical chatacterisation protocol. (a) Schematic representation of how samples were compressed at different test frequnecies using an oscilatory dynamic mechanical analysis (DMA) method; (b) schematic representation of the complex information gained from oscilatory testing, including damping and phase lag, which allows measurement of compound, storage and loss moduli and tan delta. Circled numbers in (b) correspond to the phase of compression in (a). 
Information Box 1. Overview of key terms used in deformation mechanics. Adapted from[33].

Stress - Force per unit area.

Strain - The response of a system to an applied stress, which is quantified by the amount of deformation in a material relative to its initial reference size.

Stiffness - The extent to which an object can resist external deformation.

Viscoelasticity - The term used to describe substrates that display both viscous (fluid) and elastic (solid) deformation characteristics. Viscoelastic substrates exhibit stressstiffening behaviours, meaning that the perceived/measured stiffness is dependent on the rate at which a force is applied.

Compound modulus ( $\left.\mathbf{E}^{*}\right)$ - An overall indication of stiffness, composed of a composite of the storage and loss modulus.

Storage modulus (E') - The elastic component of modulus, related to the spring constant and similar to Young's modulus. Determined by the in-phase component of the DMA cycle.

Loss modulus (E") - The viscous component of modulus due to fluid-like behaviours. Related to damping (the dissipation/loss of mechanical energy) and determined by the out-of-phase component of the DMA cycle.

Tan delta - The ratio of viscous and elastic modulus. 


\section{Histology}

Following overnight fixation in cold $4 \%(\mathrm{v} / \mathrm{v})$ paraformaldehyde, spinal cord was placed in $15 \%$ sucrose and then $30 \%(\mathrm{v} / \mathrm{v})$ sucrose. After tissue had equilibrated, sucrose was replaced with 50:50 mixture of 30\% sucrose and SurgiPath ${ }^{\mathrm{TM}}$ frozen section compound (Leica FSC22) for 4 hours at room temperature. Tissue was then placed in SurgiPath ${ }^{\mathrm{TM}}$ frozen section compound only and snap frozen in liquid nitrogen. $10 \mu \mathrm{m}$ sections were cut from along the length of the cord using a cryostat (Leica CM3050 S). These were placed on glass cover slips, gently washed, and stained with toluidine blue for bright field microscopy to visualise tissue and the presence or absence of dura.

\section{Hydrogel formulation}

Collagen hydrogels were prepared using clinical-grade type I, acid-soluble bovine telocollagen $6 \mathrm{mg} / \mathrm{mg}$ (Collagen Solutions). Collagen was first added to 10x MEM (Sigma Aldrich, M0275) in an 80:10\% v/v ratio and neutralised with $\mathrm{NaOH}$. The solution was then mixed and a further $10 \% \mathrm{v} / \mathrm{v}$ DMEM/F-12 GlutaMax $^{\mathrm{TM}}$ cell culture media added. Where collagen was diluted, $0.1 \mathrm{M}$ acetic acid was added prior to reconstitution with other reagents.

The components of fibrin hydrogels were sourced from clinical-grade surgical fibrin sealant (TISSEEL ${ }^{\mathrm{TM}}$, Baxter). Fibrinogen and thrombin components were reconstituted according to manufacturer instructions. Fibrinogen stock solution was diluted as $\mathrm{v} / \mathrm{v} /$ in DMEM/F-12 GlutaMax ${ }^{\mathrm{TM}}$ to generate different hydrogel formulations, whilst thrombin stock solution was diluted 1:4 to encourage hydrogel formation over the formation of surgical glue/sealant. Modified fibrinogen and thrombin stocks were then mixed at the time of gelation. For both collagen and fibrin hydrogels $500 \mu \mathrm{L}$ gel solution was plated into 24-well tissue culture plates. Gels were left to set for 25 minutes on a plate heater at $37.5^{\circ} \mathrm{C}$ and 
$1 \mathrm{~mL}$ fresh media was then added. Gels were placed in a cell culture incubator (humidified, $37^{\circ} \mathrm{C}, 5 \% \mathrm{CO}_{2}$ ) for a further 1 hour before mechanical testing was performed. Care was taken to avoid the formation of bubbles at all stages in the gel making process.

GMP-compatible sodium alginate (Novamatrix) was dissolved in DMEM or Neurobasal media under sterile conditions to form final concentrations of either $1.0 \%$ or $1.5 \% \mathrm{w} / \mathrm{v}$. To make gels, $100 \mu \mathrm{L}$ of alginate solution was placed inside a ThinCert ${ }^{\mathrm{TM}}$ in a 24-well plate. $1 \mathrm{~mL}$ of $102 \mathrm{mM}$ calcium chloride $\left(\mathrm{CaCl}_{2}\right.$, Sigma Aldrich) was then added into the well to facilitate crosslinking. After gelation, $\mathrm{CaCl}_{2}$ was removed and hydrogels were washed twice with $0.9 \% \mathrm{w} / \mathrm{v}$ saline. Hydrogels were then placed in cell culture media for a further 1 hour prior to mechanical testing.

\section{Statistical testing}

IBM SPSS Statistics 22.0 (IBM Inc. Armonk, NY) was used for all statistical analysis. Statistical tests were assumed to be significant at the 5\% level. Where appropriate, data were assessed for normality and Levene's statistic was used to assess for equality of variance. 


\section{Results}

\section{Ex vivo spinal cord tissue becomes softer with increased time post-mortem}

Using a previously published syringe expulsion method[34], spinal cord tissue was extracted from the spinal column devoid of dura or accompanying connective tissue (Figure 2a). This prevented lengthy dissection of the spinal musculature and vertebra, allowing spinal cord tissue to be rapidly extracted and tested postmortem. Different anatomical regions of the spinal cord were then used to assess the effect of time post-mortem on the mechanical properties of fresh CNS tissue (Figure 2b). All regions of spinal cord followed a similar frequency-dependent response, whereby compound modulus values increased with higher frequencies (Figure 2c, Figure 2d and Figure 2e). However, even when transported in specialised transplantation buffer, the mechanical properties of spinal cord tissue changed significantly with time post-mortem (Figure $2 \mathrm{f}$ ). As time between dissection and testing increased, the stiffness of fresh spinal cord tissue decreased. 40 minutes was the fastest time in which the spinal cord could feasibly be dissected, transported, geometrically measured and mechanically tested after death, and was therefore the time chosen for subsequent experiments. Compound modulus values were reduced when spinal cord was tested at 50 and 60 minutes post-mortem and did not recover to the modulus values measured at 40 minutes. There were no significant differences in compound modulus values between tissue tested at 50 and 60 minutes post-mortem; however, the viscoelastic profile of tissue differed between all of the time conditions tested (Figure 2g). Elastic behaviours (storage modulus E') were more dominant when spinal cord was tested at 40 minutes post-mortem (Figure 2c), as indicated by lower tan delta values, whilst at 50 and 60 minutes viscous behaviours (loss modulus E") became more dominant (Figure $2 \mathrm{~d}$ and Figure 2e). A particular tapering of the viscous response was observed at higher strain rates in spinal cord tissue tested at 50 
minutes post-mortem (Figure 2d). Differences were detected between tissue tested at 40 minutes and 50 or 60 minutes, both for modulus values measured at low strain rates such as $1 \mathrm{~Hz}$ and the response of tan delta to increasing strain rates. The tan delta values for spinal cord tissue tested at 40 mins were lower, which indicated that the overall elastic component of tissue modulus is lost as tissue degrades with increasing time post-mortem. 
(a)
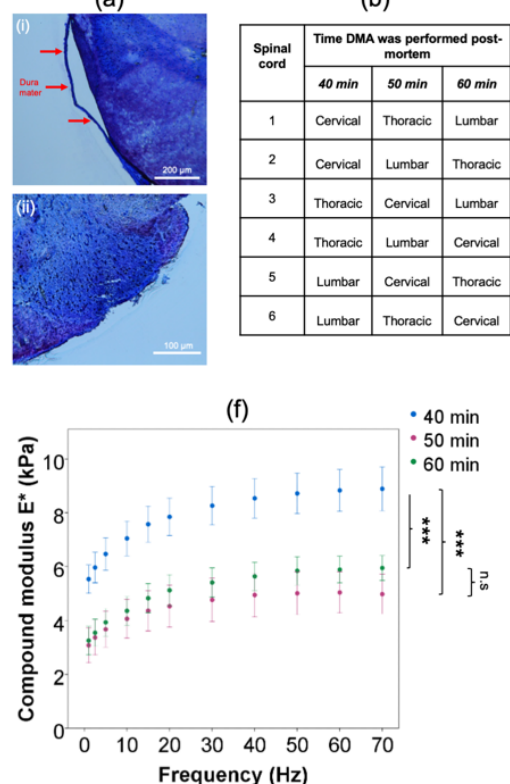

(g)

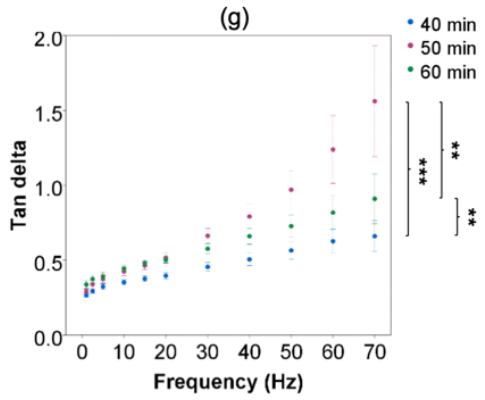

(c)

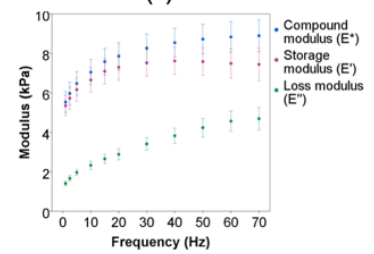

(d)

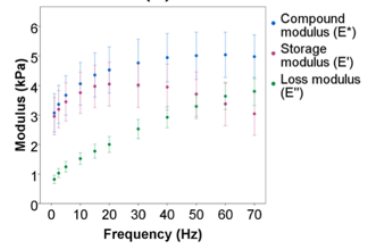

(e)

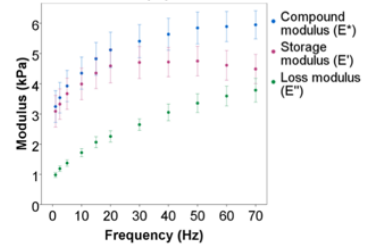

Figure 2. Effect of time post-mortem on changes to the bulk mechanical properties of fresh ex vivo spinal cord tissue. (a) Validation of rapid syringe expulsion technique to remove spinal cord tissue, where top panel displays spinal cord extracted using open dissection and bottom panel displays cord tissue obtained using expulsion method; (b) overview of strategic testing order for different times post-mortem; (c) compound, storage and loss modulus of fresh spinal cord tissue tested at $40 \mathrm{~min}$; (d) compound, storage and loss modulus of fresh spinal cord tissue tested at 50 min; (e) compound, storage and loss modulus of fresh spinal cord tissue tested at $60 \mathrm{~min}$; (f) effect of time post-mortem on the compound modulus of fresh ex vivo spinal cord tissue; ( $\mathrm{g}$ ) effect of time post-mortem on tan delta viscoelastic ratio. All speicmens compressed radially. Means \pm SEM ( $\mathrm{n}=$ 18 samples from 6 spinal cords). Two-way ANOVA accounting for time post-mortem and frequency with Tukey post-hoc. ${ }^{* * *} \mathrm{p}<0.001,{ }^{* *} \mathrm{p}<0.01, \mathrm{n} . \mathrm{s}=$ non-significant. 


\section{Viscous properties become increasingly dominant at higher strain rates for spinal cord tissue}

For spinal cord tissue tested at 40 minutes post-mortem, cervical, thoracic and lumbar spinal cord tissue all displayed similar responses of increasing modulus to higher strain rates (Figure 3a, Figure 3b and Figure 3c). For all three anatomical regions, compound modulus values increased as strain rate (test frequency) increased. However, increases in compound modulus values were not linear. The perceived stiffness of tissue increased rapidly at strain rates between 1 and $20 \mathrm{~Hz}$, yet beyond $30 \mathrm{~Hz}$ there was limited capacity for additional stress-stiffening, with further increases becoming marginal and a gradual plateau in modulus occurring between $30-70 \mathrm{~Hz}$ (Figure 3g). Notably, at strain rates above $30 \mathrm{~Hz}$, storage modulus displayed limited capacity to increase further and there was an associated increase in loss modulus and tan delta (Figure 3d, Figure 3e and Figure 3f). At $1 \mathrm{~Hz}$, tan delta values for cervical, thoracic and lumbar spinal cord regions were similar at $0.273,0.289$ and 0.243 , respectively, and a similar trend continued up to $30 \mathrm{~Hz}$. However, beyond $30 \mathrm{~Hz}$, tan delta values for cervical spinal cord began to increase significantly compared to the corresponding values for spinal cord tissue from the thoracic and lumbar regions (Figure 3h). For instance, at 70 $\mathrm{Hz}$ cervical spinal cord had a tan delta value of 0.769 , whilst thoracic and lumbar spinal cord only increased to 0.656 and 0.638 , respectively. Overall, tan delta values for cervical spinal cord were significantly higher than other spinal cord regions, indicating a different viscoelastic response depending on the anatomical region. There were no significant differences in tan delta between thoracic and lumbar spinal cord tissue. 


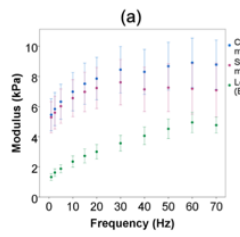

(b)

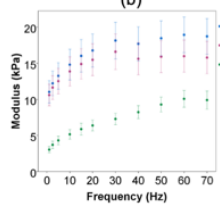

(c)

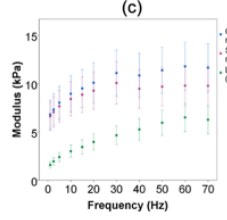

(d)

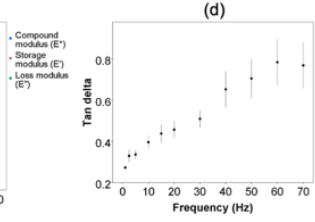

(e)
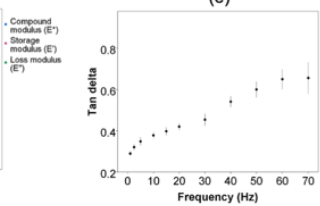

(f)

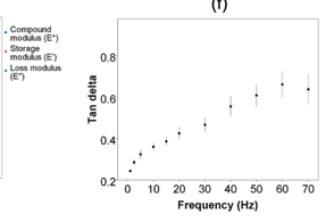

(g)
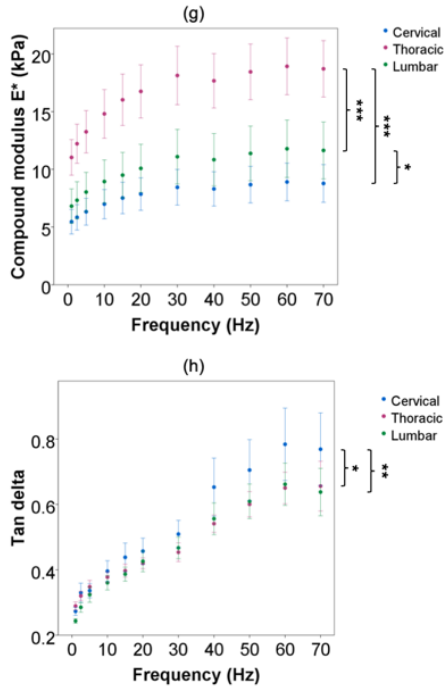

Figure 3. Bulk mechanical properties of cervical, thoracic and lumbar spinal cord tissue. (a), (b) and (c) Compound, storage and loss modulus values for cervical, thoracic and lumbar spinal cord, respectively; (d), (e) and (f) tan delta viscoelastic ratio for cervical, thoracic and lumbar spina cord, respectively; (g) comparison of compound modulus values for cervical, thoracic and lumbar spinal cord; (h) comparison of tan delta viscoelastic ratio for cervical, thoracic and lumbar spinal cord. All samples compressed radially and tested at 40 minutes post-mortem. Means \pm SEM ( $n=$ 6). Two-way ANOVA accounting for anatomical region and frequency with Tukey post-hoc. $* * * \mathrm{p}$ $<0.001, * * \mathrm{p}<0.01, * \mathrm{p}<0.05$. 


\section{Thoracic spinal cord is stiffer than cervical or lumbar spinal cord tissue}

Thoracic spinal cord tissue was found to be significantly stiffer than either cervical or lumbar spinal cord, with compound modulus values approximately double that of other spinal cord regions (Figure 3g). Increased compound modulus values for thoracic spinal cord were observed at both low and high test frequencies, suggesting that such differences were not solely due to differences in strain rate stiffening. Nevertheless, compound modulus values for thoracic cord did increase more rapidly between $1-20 \mathrm{~Hz}$, as indicated by a steeper gradient as test frequency increased (Figure $3 \mathrm{~g}$ ). This suggested that the perceived stiffness of thoracic spinal cord was highly dependent on strain rate, more so than the change observed in cervical or lumbar spinal cord. However, like cervical and lumbar spinal cord, thoracic spinal cord displayed limited capacity for further stressstiffening at test frequencies above $30 \mathrm{~Hz}$. A limited capacity for further stiffening beyond $30 \mathrm{~Hz}$ indicated that all spinal cord regions have some intrinsic mechanical similarities. A small but statistically significant difference in compound modulus was observed between cervical and lumbar spinal cord regions.

\section{Cervical spinal cord tissue displays significant mechanical anisotropy}

When compressed longitudinally, cervical spinal cord displayed a significantly different frequency-dependent response compared with radially compressed cervical spinal cord (Figure 4a). For longitudinally compressed cervical spinal cord, there was rapid stress-stiffening between 1 - $30 \mathrm{~Hz}$. However, between 30 and $40 \mathrm{~Hz}$ there was a step-wise decrease in compound modulus, followed by a plateau between $40-70 \mathrm{~Hz}$. A significant decrease in loss modulus and tan delta was also observed at $70 \mathrm{~Hz}$ (Figure $4 \mathrm{a}$ and Figure $4 \mathrm{~b}$ ). Compound modulus values for longitudinally compressed cervical spinal cord were significantly higher than radially compressed cervical spinal cord, indicating that it was approximately 
twice as stiff and that cervical spinal cord is highly mechanically anisotropic (Figure 4c). Under dynamic loading, longitudinally compressed spinal cord tissue displayed more rapid stiffening than radially compressed spinal cord, indicating a pronounced difference in how cervical spinal cord tissue responds to compressive loads depending on which direction force is applied. This relative difference in perceived stiffness was particularly marked for lower test frequencies and slower strain rates. The tan delta values of longitudinally compressed spinal cord were also generally lower than those of radially compressed spinal cord, signifying a more dominant elastic response when spinal cord was compressed parallel to its long axis and less viscous capacity than when it is compressed perpendicular to the long axis. 
(a)

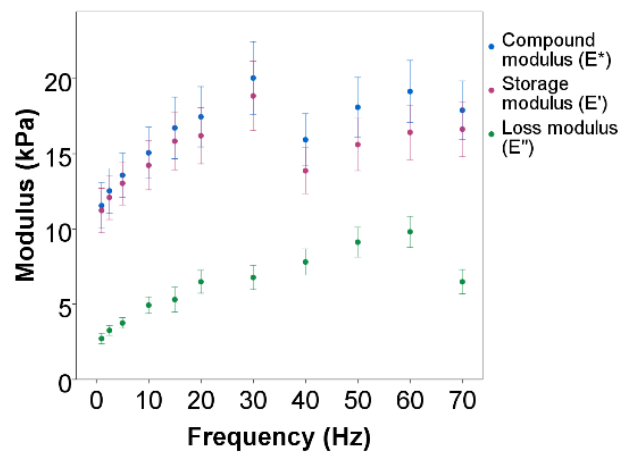

(b)

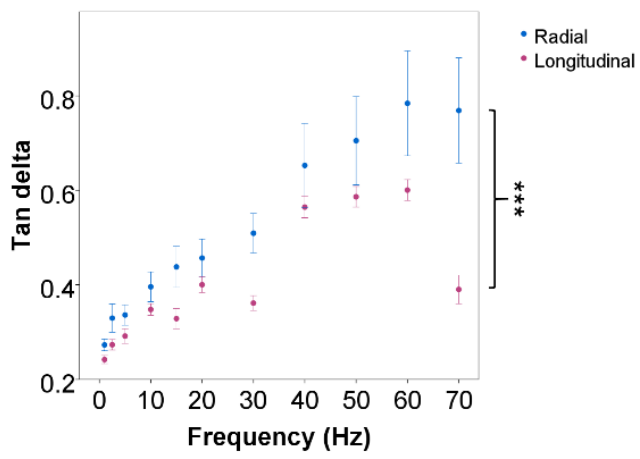

(c)
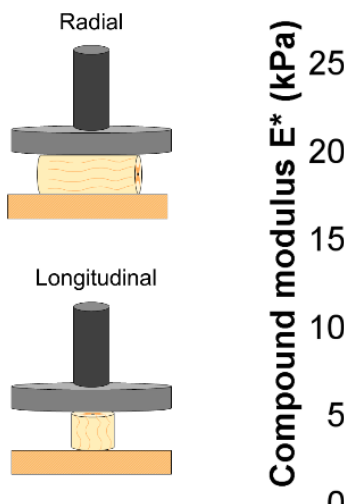

- Radial

- Longitudinal
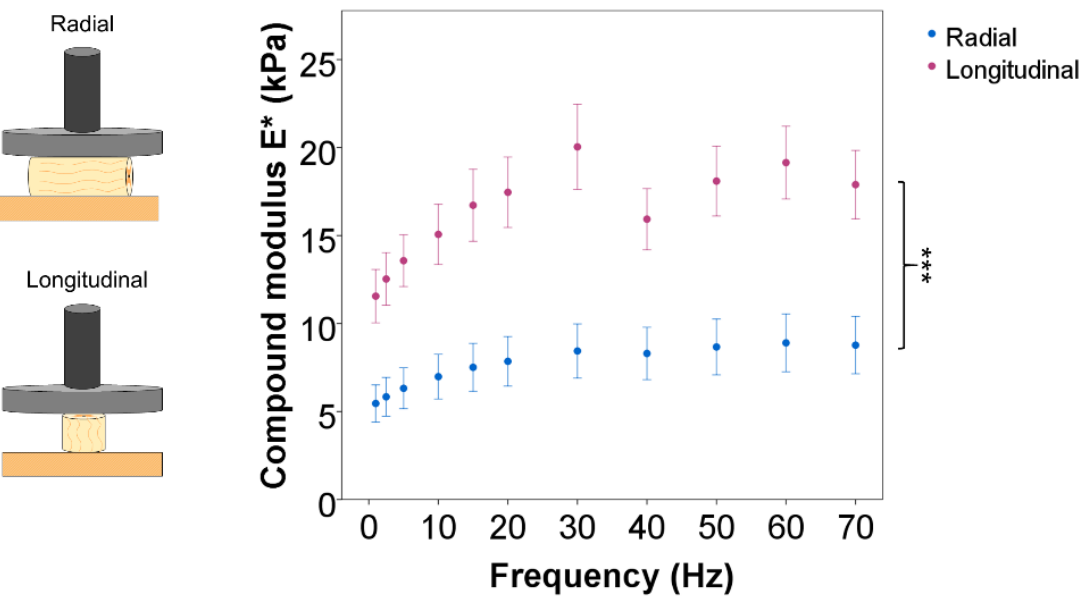

Figure 4. Mechanical anisotropy within the cervical spinal cord. (a) Compound, storage and loss modulus values for longitudinally compressed cervical spinal cord; (b) comparison of tan delta for radially and longitudinally compressed cervical spinal cord; (c) comparison of compound modulus values for radially and longitudinally compressed spinal cord. All samples tested at 40 minutes post-mortem. Means \pm SEM ( $n=6$ per orientation). Two-way ANOVA accounting for anatomical region and frequency. $* * * \mathrm{p}<0.001$. 


\section{Brain regions display significant heterogeneity in bulk mechanical properties}

The frequency-dependent response of cortex and corpus callosum brain regions was similar to radially compressed spinal cord (Figure 5a and Figure 5b).

However, significant differences in tissue stiffness were observed between cortex and corpus callosum, whereby compound modulus values for strain rates at $1 \mathrm{~Hz}$ were 4.83 and $32.15 \mathrm{kPa}$ for cortex and corpus callosum, respectively (Figure 5c). Both regions became relatively stiffer as test frequency increased, as denoted by compound modulus values of 10.21 and $63.92 \mathrm{kPa}$ at $70 \mathrm{~Hz}$ for cortex and corpus callosum, respectively. Between 40 and $70 \mathrm{~Hz}$, cortex displayed a small decrease in storage modulus, whilst corpus callosum continued to display a small but substantial increase in storage modulus (Figure 5a and Figure 5b). This was also reflected by a significant difference in tan delta between cortex and corpus callosum, with a particular divergence when higher strain rates were applied (Figure 5d). 
(a)

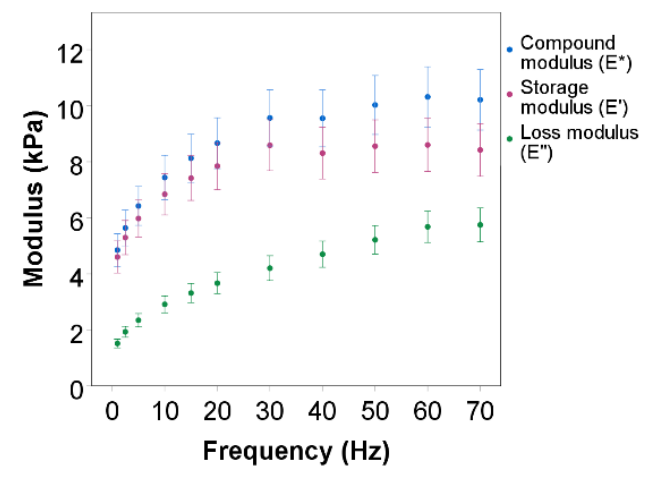

(c)

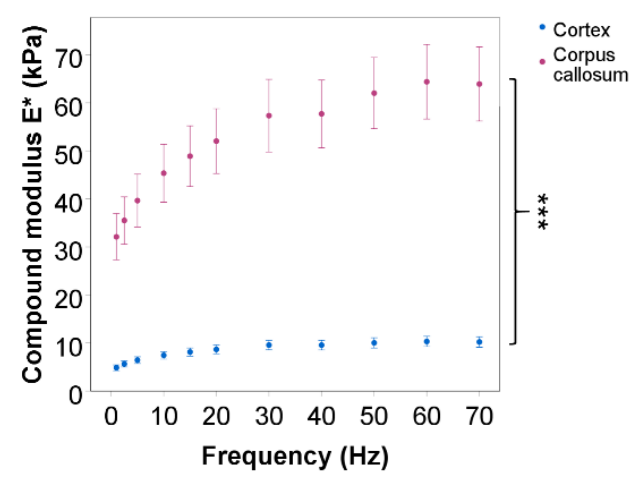

(b)

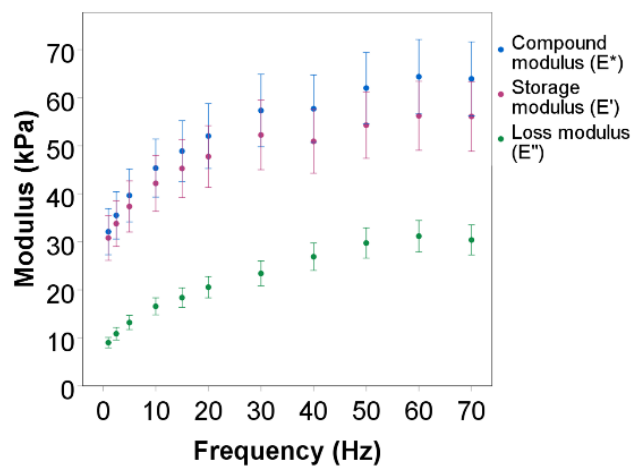

(d)

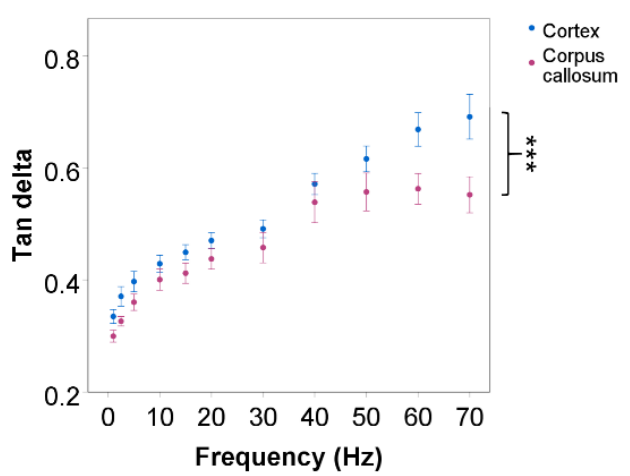

Figure 5. Regional differences in the bulk mechanical properties of brain tissue. (a) and (b) Compound, storage and loss modulus values for cortex and corpus callosum, respectively; (c) direct comparison of compound modulus between cortex and corpus callosum; (d) direct comparison of tan delta for cortex and corpus callosum. All samples tested at 40 minutes post-mortem. Means \pm SEM ( $\mathrm{n}=6$ per orientation). Two-way ANOVA accounting for anatomical region and frequency. ${ }^{* * *} \mathrm{p}<0.001$. 
Clinical-grade hydrogels can be benchmarked and tuned to mimic the stiffness properties of specific brain and spinal cord regions for regenerative medicine

The same DMA protocol was successfully applied to measure the mechanical properties of clinical-grade hydrogels suitable for use in regenerative medicine. The protocol displayed appropriate sensitivity to distinguish subtle differences in modulus values between collagen, fibrin and alginate hydrogels of different formulations. For example, $3 \mathrm{mg} / \mathrm{mL}$ collagen was found to be approximately twice as soft as $6 \mathrm{mg} / \mathrm{mL}$ collagen, and $10 \% \mathrm{v} / \mathrm{v}$ fibrin was softer than $20 \% \mathrm{v} / \mathrm{v}$ fibrin (Figure 6a, Figure 6b, Figure $6 \mathrm{c}$ and Figure $6 \mathrm{~d}$ ). However, in addition to detecting differences in modulus value, the mechanical testing protocol was also able to detect small variations in how different hydrogels responded to increasing strain rates. Collagen hydrogels displayed a pronounced increase in tan delta values at higher test frequencies, indicating a dominance of viscous rather than elastic behaviours at faster strain rates (Figure 6a and Figure 6b). In contrast, tan delta values for fibrin hydrogels increased linearly in a frequency-dependent manner (Figure 6c and Figure 6d), suggesting a more consistent elastic (storage modulus) response at higher strain rates. Alginate hydrogels displayed the opposite trend with a largely plateaued tan delta response (Figure 6e and Figure 6f), indicating a relative inability to stiffen in response to increasing strain rate and suggesting almost exclusively elastic behaviour.

Direct benchmarking of hydrogel mechanical properties against fresh spinal cord tissue indicated that $6 \mathrm{mg} / \mathrm{mL}$ collagen hydrogels closely approximated the stiffness of cervical spinal cord (Figure 6g). Yet, the viscoelastic response of cervical spinal cord tissue, particularly at lower strain rates, was more closely approximated by $20 \%$ fibrin hydrogels (Figure $6 \mathrm{~h}$ ). This provides proof of principle that the mechanical measurement protocol had the appropriate 
sensitivity to detect subtle differences in both biomaterial stiffness and viscoelastic response and map this directly against spinal cord or brain tissue. By increasing or decreasing the relative concentration of collagen, fibrin or alginate within hydrogels it was possible to alter the mechanical properties of materials. For example, the compound modulus values of the materials increased with increasing concentration (Figure 6a-f) However, it is worth noting that, even when hydrogels composed of different substrates were tuned to have similar modulus values, the response of hydrogels to increasing strain rates and the extent of frequency-dependent stiffening was different (Figure 6g and Figure 6h). This suggests that simply matching the stiffness of biomaterials to the tissue of interest may not be sufficient to ensure fully optimised mechanical properties. 

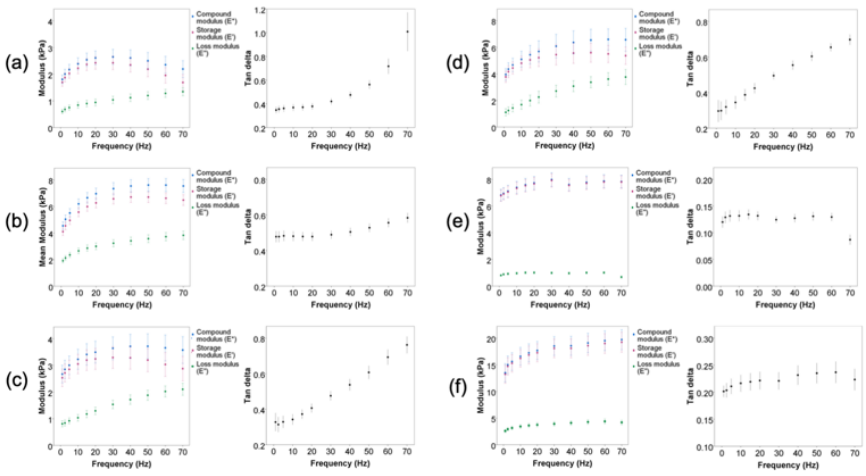

(g)
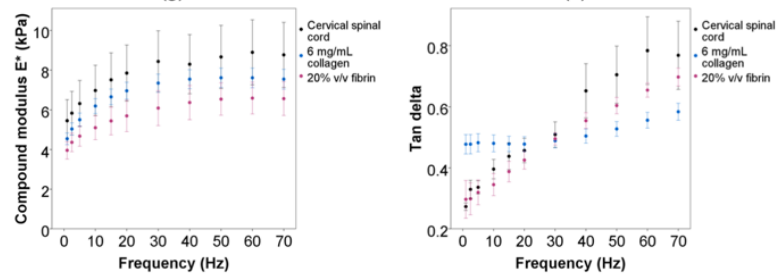

(i)

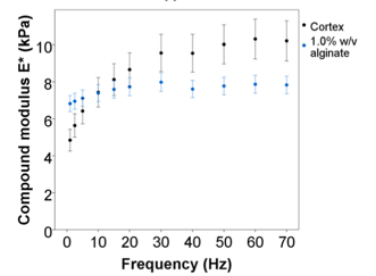

(j)

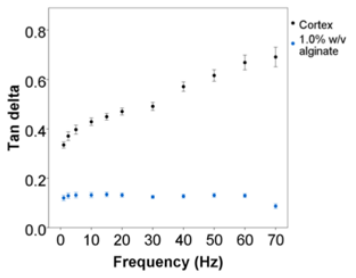

Figure 6. Comparison of clinical-grade biomaterials against spinal cord and brain tissue for regenerative medicine applications. (a) $3 \mathrm{mg} / \mathrm{mL}$ collagen hydrogel; (b) 6 $\mathrm{mg} / \mathrm{mL}$ collagen hydrogel; (c) $10 \% \mathrm{v} / \mathrm{v}$ fibrin hydrogel; (d) $20 \% \mathrm{v} / \mathrm{v}$ fibrin hydrogel; (e) $1.0 \% \mathrm{w} / \mathrm{v}$ alginate; (f) $1.5 \% \mathrm{w} / \mathrm{e}$ alginate; (g) mechanical benchmarking of collagen and fibrin hydrogels against cervical spinal cord compound modulus; (h) mechanical benchmarking of collagen and fibrin hydrogels against cervical spinal cord tan delta (i) mechanical benchmarking of alginate against brain cortex compound modulus; (j) mechanical benchmarking of alginate against brain cortex tan delta. Means $\pm \operatorname{SEM}(n=12$ collagen gels per condition across 4 unique gel batches, $\mathrm{n}=6$ fibrin gels per condition from 3 unique gel batches, $n=6$ alginate gels per condition from 3 unique gel batches, $n=6$ cervical spinal cord, $\mathrm{n}=6$ brain cortex). 


\section{Discussion}

A bespoke mechanical characterisation protocol was successfully developed and used to characterise the bulk mechanical properties of spinal cord, brain and clinical-grade biomaterials suitable for use in regenerative medicine. It facilitated robust measurement, displaying appropriate sensitivity to detect subtle differences in tissue stiffness and viscoelastic characteristics at physiologically relevant strain[36]. This enabled sophisticated like-for-like comparison of clinical-grade biomaterials against CNS tissue, enabling mechanical 'benchmarking' and offering a new tool for regenerative medicine applications.

Spinal cord and brain tissue responded non-linearly to increasing strain rates, indicating viscoelastic behaviours similar to those reported previously[37, 38]. However, this study identified that even when stored and transported in specialised tissue preservation buffer, the mechanical properties of CNS tissue changed rapidly within the first hour post-mortem. In contrast to previously published reports, some of which have tested tissue up to 60 hours postmortem[38, 39], this protocol allowed the rapid characterisation of tissue within 40 minutes of death. Upon dissection, rapid characterisation of CNS tissue is essential for ensuring that mechanical properties measured reflect true values, and previous reports have suggested significant changes to mechanical properties with increasing time post-mortem[33, 40]. This testing protocol also did not require any substantial tissue processing, where reagents or preparation steps used in some other measurement techniques have the potential to modify tissue mechanical properties or cause deviation from the values found in fresh tissue[41]. However, even when using optimised transportation and storage conditions, it is possible that a loss of perfusion pressure may cause differences in mechanical properties between fresh ex vivo tissue and CNS tissue in situ[42, 43]. 
The mechanical properties of the CNS tissues were found to vary significantly by anatomical region. Thoracic spinal cord was significantly stiffer than cervical or lumbar spinal cord, although it displayed similar viscoelastic behaviours in response to changes in strain rate. This corroborates previous reports, which suggest that thoracic spinal cord may respond differently to cervical or lumbar spinal cord when external strain is applied and displacement is measured over time[39]. Indeed, in the context of tissue engineered therapies for spinal cord repair, it will be important to consider that different regions of the spinal cord may be require biomaterials with different mechanical properties. For example, biomaterials used in repair of the thoracic spinal cord may need to be relatively stiffer than those implanted into the cervical spinal cord. The viscoelastic properties of spinal cord tissue were also found to vary by anatomical region. Tan delta values suggested that cervical spinal cord responded differently to thoracic or lumbar spinal cord at higher strain rates, where cervical spinal cord displayed a greater propensity for viscous rather than elastic behaviour. This may reflect a physiological adaptation, as the human spinal cord has been reported to stretch the most between spinal levels C2-T1 during normal movement[44]. The reduced elastic capacity of cervical spinal cord tissue at higher strain rates may also provide insights into why the cervical spinal cord is the most common site of injury after traumatic SCI[45].

Cervical spinal cord tissue displayed significant mechanical anisotropy, both in terms of tissue stiffness and the response of tissue to varying strain rates. When compressed parallel to its long axis (longitudinally) cervical spinal cord was not only stiffer, but strain-stiffened much more rapidly. This suggests that there are differences in the tissue response depending on the direction in which compressive forces are applied. A step-wise decrease in modulus midway through 
the frequency sweep cycle also indicated that longitudinally compressed spinal cord was unable to accommodate higher strains, suggesting that the tissue may have mechanically failed or been irreversibly altered. Mechanical anisotropy in the human spinal cord has been previously linked to the microstructural properties of anisotropy within white matter tissue[46, 47]. An improved understanding of the mechanical anisotropy that exists within the spinal cord is important for generating more accurate in silico models of traumatic spinal cord injury[48].

Cortex and corpus callosum were tested to assess for mechanical differences between predominant grey and white matter regions of the brain, respectively[49]. White matter tissue derived from the corpus callosum was found to be significantly stiffer than cortex and compound modulus values also increased much more rapidly in response to increasing strain rate. Cortex and corpus callosum tissue responded differently, and corpus callosum displayed a proportionally more elastic response, particularly at strain rates above $50 \mathrm{~Hz}$. Previous studies using bovine brain found the bulk mechanical properties of white matter to be 39\% stiffer than grey matter brain tissue[50]; however, direct comparisons are problematic due to different times of mechanical testing postmortem and species. This may suggest that the relative stiffness of white matter tissue becomes less pronounced as more time elapses post-mortem, and indeed some studies using different methodologies have observed grey matter tissue to be stiffer when using AFM to obtain topographical surface measurements, although results remain contradictory[51]. Relative differences in the stiffness of grey and white matter tissue have also been shown to become more pronounced when larger strains or faster strain rates are applied[52, 53].

Collagen, fibrin and alginate hydrogels compliant with good-manufacturing practice (GMP) production and suitable for use in regenerative medicine and 
tissue engineering were also mechanically characterised. Subtle differences in the stiffness and the viscoelastic response of similar material formulations could be distinguished clearly. For example, even when materials displayed similar compound modulus values, differences in tan delta highlighted variations in viscoelastic behaviour. By generating a unique mechanical profile for each biomaterial it was possible to perform more comprehensive comparisons than have been previously reported. Direct and robust comparisons between biomaterials and spinal cord or brain tissue are something that has previously been difficult to achieve, owing to different measurement techniques being required for the characterisation of biomaterials and CNS tissue. By enabling direct mechanical benchmarking, it was possible to precisely compare both the stiffness and viscoelastic response of each biomaterial to CNS tissue. For example, whilst $1.0 \% \mathrm{w} / \mathrm{v}$ alginate displayed similar stiffness values to brain cortex, the viscoelastic response to deformation at higher frequencies was notably different. Likewise, $6 \mathrm{mg} / \mathrm{mL}$ collagen closely matched cervical spinal cord stiffness, yet $20 \% \mathrm{v} / \mathrm{v}$ fibrin better approximated the overall viscoelastic response and tan delta values. Compressive modulus values are also a more useful metric than the tensile or shear modulus values provided by some other methodologies, as it is compressive forces that are likely to dominate when biomaterials are implanted into the CNS. We therefore propose that this specific DMA protocol will facilitate more sophisticated comparisons of biomaterial mechanical properties with CNS tissue than has previously been reported, enabling a new generation of stiffness-matched biomaterials specifically tuned for CNS regenerative medicine.

Beyond regenerative medicine, there is also a fundamental need for improved understanding of the mechanical properties of CNS tissue, as changes to tissue mechanical properties have been implicated in a range of pathologies including 
multiple sclerosis, traumatic brain injury and Alzheimer's disease[54-57]. Additionally, biophysical cues and mechanotransduction offer novel therapeutic strategies outside the realm of traditional soluble factor or drug-based approaches. Notably, the mechanical properties of the extracellular environment have been implicated in the invasiveness and spread of tumours such as glioblastoma throughout the brain[58]. This premise has been exploited to lure traditionally inoperable intracranial tumour cells out of the brain and into cytotoxic hydrogels[59], and the Tumour Monorail ${ }^{\mathrm{TM}}$ has recently been awarded FDA 'Breakthrough Device' status. This study provides a new tool for the mechanical benchmarking of such hydrogels, which may help increase understanding of the effect of stiffness gradients on tumour cell migration and allow for further refinement of future biophysical therapies.

One potential limitation of this study was that samples were tested at room temperature $\left(21^{\circ} \mathrm{C}\right)$ whereas at $37^{\circ} \mathrm{C}$, the modulus values for hydrogels may differ slightly. For instance, a 16\% increase in the shear modulus of alginate gels tested at $23{ }^{\circ} \mathrm{C}$ and $37^{\circ} \mathrm{C}$ has been reported [60]. The effect of temperature on tissue and hydrogels stiffness remains poorly characterised, so all values reported in this study were measured under controlled room temperature conditions to reduce variability. Furthermore, a previous study showed that the difference between in vitro measurements and in vivo mechanical properties of porcine brain tissue can be minimised if tests are conducted at room temperature and specimens have been previously preserved at ice-cold temperatures [61]. Another important consideration is that this study used fresh tissue from healthy animals whereas biomaterial therapies would be deployed after injury where mechanical properties may vary and change over time. The methodology used here could be applied in future studies to explore this, perhaps enabling the mechanical properties of biomaterial interventions to match specific damage environments. 
In the current study, the comparison between tissue samples and hydrogels was performed via DMA at a fixed $2 \%$ strain rate with a frequency sweep. This protocol was chosen for pragmatic reasons as it eliminates irregularities and irreversible damage to samples. Moreover, it allows for robust mechanical testing to be executed relatively quickly, thereby reducing the risk of changes due to increasing time post-mortem. However, it should be noted that no single technique is likely to fully capture the complexity of the mechanical behaviours of a substrate or tissue. This is particularly important in hydrogels formed by biopolymers such as collagen and fibrin, where modulus values have been shown to vary when measuring mechanical properties under different levels of tensile and compressive strain [62] .

In conclusion, these data provide a comprehensive comparison of tissue stiffness throughout the spinal cord and brain, but also offers a pragmatic new methodology for benchmarking the mechanical properties of biomaterials for CNS repair. Future work may wish to consider applying this methodology to other soft biological tissues or materials for fundamental discovery or regenerative medicine applications. Given the mechanical anisotropy within the cervical spinal cord, it would be worthwhile developing and quantifying mechanically anisotropic biomaterials for spinal cord tissue engineering. Similarly, biomaterials designed for implantation into the brain could be tuned for specific anatomical variations of mechanical properties. 


\section{AUTHOR CONTRIBUTIONS:}

RDB led in the development of the DMA protocol and performed all the work for spinal cord tissue and collagen and fibrin hydrogels. RDB also performed the dissection and isolation of fresh brain tissue, along with the majority of data analysis and writing of the manuscript. RE was responsible for microdissection of the brain and isolation of cortex and corpus callosum, along with synthesis of alginate hydrogels. DE performed mechanical testing of brain tissue and alginate hydrogels. DC and JBP provided senior input and guidance throughout. All authors contributed intellectually to the design of experiments and approved the final version of this manuscript prior to submission for publication.

\section{ROLE OF THE FUNDING SOURCE:}

$\mathrm{RDB}$ is kindly funded by The Sackler Fund (MB/PhD award) and RE receives funding from EPSRC grant EP/L01646X.

\section{CONFLICT OF INTERESTS:}

None of the authors have any relevant conflicts of interest to declare.

\section{ETHICS COMMITTEE APPROVAL:}

Not applicable.

\section{REFERENCES:}


[1] J.H. Chin, N. Vora, The global burden of neurologic diseases, Neurology 83(4) (2014) 349-351.

[2] World Health Organization, Spinal cord injury, 2013.

http://www.who.int/mediacentre/factsheets/fs384/en/. (Accessed 30/09/2015.

[3] Unite 2 Fight Paralysis, The Economics of Spinal Cord Injury 2004.

[4] Neurodegenerative diseases raise profound questions, BMJ 324(7352) (2002) $0-0$.

[5] T. Führmann, M.S. Shoichet, The role of biomaterials in overcoming barriers to regeneration in the central nervous system, Biomedical Materials 13(5) (2018) 050201.

[6] Y.-S. Chen, H.-J. Harn, T.-W. Chiou, The Role of Biomaterials in Implantation for Central Nervous System Injury, Cell Transplant 27(3) (2018) 407-422.

[7] S. Liu, T. Schackel, N. Weidner, R. Puttagunta, Biomaterial-Supported Cell Transplantation Treatments for Spinal Cord Injury: Challenges and Perspectives, Frontiers in Cellular Neuroscience 11(430) (2018).

[8] A.M. Ziemba, R.J. Gilbert, Biomaterials for Local, Controlled Drug Delivery to the Injured Spinal Cord, Frontiers in pharmacology 8 (2017) 245.

[9] L. Yao, W. Daly, B. Newland, S. Yao, W. Wang, B.K. Chen, N. Madigan, A. Windebank, A. Pandit, Improved axonal regeneration of transected spinal cord mediated by multichannel collagen conduits functionalized with neurotrophin-3 gene, Gene therapy 20(12) (2013) 1149-57.

[10] B. Chen, Z. Xiao, Y. Zhao, J. Dai, Functional biomaterial-based regenerative microenvironment for spinal cord injury repair, National Science Review 4(4) (2017) 530-532.

[11] A. Tuladhar, S.L. Payne, M.S. Shoichet, Harnessing the Potential of Biomaterials for Brain Repair after Stroke, Frontiers in Materials 5(14) (2018).

[12] F.L. Maclean, M.K. Horne, R.J. Williams, D.R. Nisbet, Review: Biomaterial systems to resolve brain inflammation after traumatic injury, APL bioengineering 2(2) (2018) 021502.

[13] D. Hadavi, A.A. Poot, Biomaterials for the Treatment of Alzheimer's Disease, Front Bioeng Biotechnol 4 (2016) 49-49.

[14] N. Moriarty, E. Dowd, Brain repair for Parkinson's disease: is the answer in the matrix?, Neural Regen Res 13(7) (2018) 1187-1188.

[15] M. Pekny, M. Pekna, Astrocyte Reactivity and Reactive Astrogliosis: Costs and Benefits, Physiological Reviews 94(4) (2014) 1077-1098.

[16] A.R. Clark, A.B. Carter, L.E. Hager, E.M. Price, In Vivo Neural Tissue Engineering: Cylindrical Biocompatible Hydrogels That Create New Neural Tracts in the Adult Mammalian Brain, Stem cells and development 25(15) (2016) 1109-18. 
[17] E. Herran, J.A. Ruiz-Ortega, A. Aristieta, M. Igartua, C. Requejo, J.V. Lafuente, L. Ugedo, J.L. Pedraz, R.M. Hernandez, In vivo administration of VEGF- and GDNF-releasing biodegradable polymeric microspheres in a severe lesion model of Parkinson's disease, European journal of pharmaceutics and biopharmaceutics : official journal of Arbeitsgemeinschaft fur Pharmazeutische Verfahrenstechnik e.V 85(3 Pt B) (2013) 1183-90.

[18] M. Pekny, M. Pekna, Astrocyte reactivity and reactive astrogliosis: costs and benefits, Physiological reviews 94(4) (2014) 1077-98.

[19] P. Moshayedi, F. Costa Lda, A. Christ, S.P. Lacour, J. Fawcett, J. Guck, K. Franze, Mechanosensitivity of astrocytes on optimized polyacrylamide gels analyzed by quantitative morphometry, Journal of physics. Condensed matter : an Institute of Physics journal 22(19) (2010) 194114.

[20] P. Moshayedi, G. Ng, J.C. Kwok, G.S. Yeo, C.E. Bryant, J.W. Fawcett, K. Franze, J. Guck, The relationship between glial cell mechanosensitivity and foreign body reactions in the central nervous system, Biomaterials 35(13) (2014) 3919-25.

[21] A.J. Engler, S. Sen, H.L. Sweeney, D.E. Discher, Matrix elasticity directs stem cell lineage specification, Cell 126(4) (2006) 677-89.

[22] K.C. Clause, L.J. Liu, K. Tobita, Directed stem cell differentiation: the role of physical forces, Cell Commun Adhes 17(2) (2010) 48-54.

[23] M.G. Haugh, T.J. Vaughan, C.M. Madl, R.M. Raftery, L.M. McNamara, F.J. O'Brien, S.C. Heilshorn, Investigating the interplay between substrate stiffness and ligand chemistry in directing mesenchymal stem cell differentiation within 3D macro-porous substrates, Biomaterials 171 (2018) 23-33. [24] W.J. Hadden, J.L. Young, A.W. Holle, M.L. McFetridge, D.Y. Kim, P. Wijesinghe, H. Taylor-Weiner, J.H. Wen, A.R. Lee, K. Bieback, B.N. Vo, D.D. Sampson, B.F. Kennedy, J.P. Spatz, A.J. Engler, Y.S. Choi, Stem cell migration and mechanotransduction on linear stiffness gradient hydrogels, Proc Natl Acad Sci U S A 114(22) (2017) 5647-5652.

[25] A.P. Balgude, X. Yu, A. Szymanski, R.V. Bellamkonda, Agarose gel stiffness determines rate of DRG neurite extension in 3D cultures, Biomaterials 22(10) (2001) 1077-84.

[26] L.A. Flanagan, Y.-E. Ju, B. Marg, M. Osterfield, P.A. Janmey, Neurite branching on deformable substrates, Neuroreport 13(18) (2002) 2411-2415. [27] D. Koch, W.J. Rosoff, J. Jiang, H.M. Geller, J.S. Urbach, Strength in the periphery: growth cone biomechanics and substrate rigidity response in peripheral and central nervous system neurons, Biophys J 102(3) (2012) 452-460. [28] C.A. Grant, P.C. Twigg, D.J. Tobin, Static and dynamic nanomechanical properties of human skin tissue using atomic force microscopy: effect of scarring in the upper dermis, Acta Biomater 8(11) (2012) 4123-9. 
[29] B. Hinz, Tissue stiffness, latent TGF-beta1 activation, and mechanical signal transduction: implications for the pathogenesis and treatment of fibrosis, Curr Rheumatol Rep 11(2) (2009) 120-6.

[30] E. Moeendarbary, I.P. Weber, G.K. Sheridan, D.E. Koser, S. Soleman, B. Haenzi, E.J. Bradbury, J. Fawcett, K. Franze, The soft mechanical signature of glial scars in the central nervous system, Nat Commun 8 (2017) 14787.

[31] T. Saxena, J. Gilbert, D. Stelzner, J. Hasenwinkel, Mechanical characterization of the injured spinal cord after lateral spinal hemisection injury in the rat, J Neurotrauma 29(9) (2012) 1747-57.

[32] J.G. Cooper, D. Sicard, S. Sharma, S. Van Gulden, T.L. McGuire, M.P. Cajiao, D.J. Tschumperlin, J.A. Kessler, Spinal Cord Injury Results in Chronic Mechanical Stiffening, J Neurotrauma 37(3) (2020) 494-506.

[33] R.D. Bartlett, D. Choi, J.B. Phillips, Biomechanical properties of the spinal cord: implications for tissue engineering and clinical translation, Regenerative medicine 11(7) (2016) 659-73.

[34] H.S. Kennedy, C. Jones, P. Caplazi, Comparison of standard laminectomy with an optimized ejection method for the removal of spinal cords from rats and mice, Journal of Histotechnology 36(3) (2013) 86-91.

[35] S. Cheng, E.C. Clarke, L.E. Bilston, The effects of preconditioning strain on measured tissue properties, Journal of biomechanics 42(9) (2009) 1360-2.

[36] J. Scifert, K. Totoribe, V. Goel, J. Huntzinger, Spinal cord mechanics during flexion and extension of the cervical spine: a finite element study, Pain Physician 5(4) (2002) 394-400.

[37] S.S. Shetye, K.L. Troyer, F. Streijger, J.H.T. Lee, B.K. Kwon, P.A. Cripton, C.M. Puttlitz, Nonlinear viscoelastic characterization of the porcine spinal cord, Acta biomaterialia 10(2) (2014) 792-797.

[38] S. Budday, G. Sommer, G.A. Holzapfel, P. Steinmann, E. Kuhl, Viscoelastic parameter identification of human brain tissue, Journal of the Mechanical Behavior of Biomedical Materials 74 (2017) 463-476.

[39] R.J. Fiford, L.E. Bilston, The mechanical properties of rat spinal cord in vitro, Journal of Biomechanics 38(7) (2005) 1509-1515.

[40] R.J. Oakland, R.M. Hall, R.K. Wilcox, D.C. Barton, The biomechanical response of spinal cord tissue to uniaxial loading, Proceedings of the Institution of Mechanical Engineers. Part H, Journal of engineering in medicine 220(4) (2006) 489-92.

[41] J.T. Morgan, V.K. Raghunathan, S.M. Thomasy, C.J. Murphy, P. Russell, Robust and artifact-free mounting of tissue samples for atomic force microscopy, Biotechniques 56(1) (2014) 40-42.

[42] H. Metz, J. McElhaney, A.K. Ommaya, A comparison of the elasticity of live, dead, and fixed brain tissue, Journal of Biomechanics 3(4) (1970) 453-458. 
[43] C.D. Etz, G. Di Luozzo, S. Zoli, R. Lazala, K.A. Plestis, C.A. Bodian, R.B. Griepp, Direct Spinal Cord Perfusion Pressure Monitoring in Extensive Distal Aortic Aneurysm Repair, The Annals of Thoracic Surgery 87(6) (2009) 17641774.

[44] J.D. Reid, Effects of flexion-extension movements of the head and spine upon the spinal cord and nerve roots, Journal of neurology, neurosurgery, and psychiatry 23 (1960) 214-21.

[45] A. Nulle, U. Tjurina, R. Erts, A. Vetra, A profile of traumatic spinal cord injury and medical complications in Latvia, Spinal Cord Series and Cases 3(1) (2017) 17088.

[46] H. Mamata, F.A. Jolesz, S.E. Maier, Characterization of central nervous system structures by magnetic resonance diffusion anisotropy, Neurochemistry International 45(4) (2004) 553-560.

[47] R.W. Carlsen, N.P. Daphalapurkar, The importance of structural anisotropy in computational models of traumatic brain injury, Front Neurol 6 (2015) 28. [48] L. Fradet, P.-J. Arnoux, V. Callot, Y. Petit, Geometrical variations in white and gray matter affect the biomechanics of spinal cord injuries more than the arachnoid space, Advances in Mechanical Engineering 8(8) (2016) 1687814016664703.

[49] A. Fitsiori, D. Nguyen, A. Karentzos, J. Delavelle, M.I. Vargas, The corpus callosum: white matter or terra incognita, Br J Radiol 84(997) (2011) 5-18. [50] S. Budday, R. Nay, R. de Rooij, P. Steinmann, T. Wyrobek, T.C. Ovaert, E. Kuhl, Mechanical properties of gray and white matter brain tissue by indentation, Journal of the mechanical behavior of biomedical materials 46 (2015) 318-330. [51] D.E. Koser, E. Moeendarbary, J. Hanne, S. Kuerten, K. Franze, CNS cell distribution and axon orientation determine local spinal cord mechanical properties, Biophys J 108(9) (2015) 2137-47.

[52] K. Ichihara, T. Taguchi, Y. Shimada, I. Sakuramoto, S. Kawano, S. Kawai, Gray matter of the bovine cervical spinal cord is mechanically more rigid and fragile than the white matter, Journal of neurotrauma 18(3) (2001) 361-7. [53] Y. Feng, E.H. Clayton, Y. Chang, R.J. Okamoto, P.V. Bayly, Viscoelastic properties of the ferret brain measured in vivo at multiple frequencies by magnetic resonance elastography, J Biomech 46(5) (2013) 863-70.

[54] K.J. Streitberger, I. Sack, D. Krefting, C. Pfuller, J. Braun, F. Paul, J.

Wuerfel, Brain viscoelasticity alteration in chronic-progressive multiple sclerosis, PloS one 7(1) (2012) e29888.

[55] T. Boulet, M.L. Kelso, S.F. Othman, Long-term in vivo imaging of viscoelastic properties of the mouse brain after controlled cortical impact, Journal of neurotrauma 30(17) (2013) 1512-20. 
[56] R. Paparcone, M.A. Pires, M.J. Buehler, Mutations alter the geometry and mechanical properties of Alzheimer's Abeta(1-40) amyloid fibrils, Biochemistry 49(41) (2010) 8967-77.

[57] M.C. Murphy, D.T. Jones, C.R. Jack, Jr., K.J. Glaser, M.L. Senjem, A. Manduca, J.P. Felmlee, R.E. Carter, R.L. Ehman, J. Huston, 3rd, Regional brain stiffness changes across the Alzheimer's disease spectrum, Neuroimage Clin 10 (2015) 283-290.

[58] T.J. Grundy, E. De Leon, K.R. Griffin, B.W. Stringer, B.W. Day, B. Fabry, J. Cooper-White, G.M. O'Neill, Differential response of patient-derived primary glioblastoma cells to environmental stiffness, Scientific Reports 6 (2016) 23353. [59] A. Jain, M. Betancur, G.D. Patel, C.M. Valmikinathan, V.J. Mukhatyar, A. Vakharia, S.B. Pai, B. Brahma, T.J. MacDonald, R.V. Bellamkonda, Guiding intracortical brain tumour cells to an extracortical cytotoxic hydrogel using aligned polymeric nanofibres, Nature Materials 13 (2014) 308.

[60] C. Ganachaud, D. Bernin, D. Isaksson, K. Holmberg, An anomalous behavior of trypsin immobilized in alginate network, Applied microbiology and biotechnology 97(10) (2013) 4403-14.

[61] B. Rashid, M. Destrade, M.D. Gilchrist, Influence of preservation temperature on the measured mechanical properties of brain tissue, Journal of biomechanics 46(7) (2013) 1276-81.

[62] A.S.G. van Oosten, M. Vahabi, A.J. Licup, A. Sharma, P.A. Galie, F.C. MacKintosh, P.A. Janmey, Uncoupling shear and uniaxial elastic moduli of semiflexible biopolymer networks: compression-softening and stretch-stiffening, Scientific Reports 6(1) (2016) 19270. 